\title{
Hunting New Physics with ATLAS
}

\author{
Vasiliki A. Mitsou ${ }^{1, a}$ \\ On behalf of the ATLAS Collaboration \\ ${ }^{1}$ Instituto de Física Corpuscular (IFIC), CSIC - Universitat de València, \\ Parc Científic de la U.V., C/ Catedrático José Beltrán 2, \\ E-46980 Paterna (Valencia), Spain
}

\begin{abstract}
Highlights from recent new physics searches with the ATLAS detector at the CERN LHC are presented in this paper. They include searches for extra-dimension models, compositeness, new gauge bosons, supersymmetry, among others. Results are based on analyses of $p p$ collision data recorded at a centre-of-mass energy of $13 \mathrm{TeV}$.
\end{abstract}

\section{Introduction}

The search for phenomena beyond the Standard Model (BSM) is one of the primary motivations for the physics program at the Large Hadron Collider (LHC) [1]. The presence of dark matter in the universe and the hierarchy problem provide strong hints that new phenomena should exist. The increase of the LHC energy from 8 to $13 \mathrm{TeV}$ in 2015 greatly extended its capacity to produce new particles in $p p$ collisions. Here results from searches for exotic particles using $\sim 36 \mathrm{fb}^{-1}$ of data collected by the ATLAS detector [2] are presented, where "exotic" means BSM particles outside the framework of supersymmetry, and also not including BSM Higgs bosons (searches for such particles are reported elsewhere [3]). Other BSM searches not presented here but that are being pursued in ATLAS at $13 \mathrm{TeV}$ include, e.g., leptoquarks [4]. In addition, searches for non-standard supersymmetry, such as those involving supersymmetric partners of long lifetime are highlighted in this paper.

\section{Looking for resonances and tails in distributions}

Physics beyond the Standard Model (SM) can be probed in colliders by looking for bumps in invariantmass distributions and/or performing cut-and-count experiments expecting to observe an excess of events in their tails. Some recent searches from ATLAS are highlighted below. Specific analyses targetting signals of dark matter are described elsewhere [5].

\subsection{Dileptons}

A search has been conducted by ATLAS for new resonant and non-resonant high-mass phenomena in dielectron and dimuon final states [6]. The search uses $36.1 \mathrm{fb}^{-1}$ of proton-proton collision data,

\footnotetext{
a e-mail: vasiliki.mitsou@ific.uv.es
} 
collected at $\sqrt{s}=13 \mathrm{TeV}$ by the ATLAS experiment in 2015 and 2016. The dilepton invariant mass is used as the discriminating variable. This experimental signature benefits from a fully reconstructed final state, high signal-selection efficiency and relatively small, well-understood background, representing a powerful test for a wide range of theories beyond the SM.

The analysis is optimised for bosons, generally referred to as $Z^{\prime}$, which would manifest as a narrow resonance through its decay, in the dilepton mass spectrum. These heavy spin-1 bosons are predicted in models with extended gauge groups which feature additional U(1) symmetries. This search is also sensitive to a series of other models that predict the presence of narrow dilepton resonances: the Randall-Sundrum (RS) model with a warped extra dimension giving rise to spin-2 graviton excitations [7], the quantum black-hole model [8], the $Z^{*}$ model [9], and the minimal walking technicolour model [10]. Moreover, non-resonant phenomena are probed: effective four-fermion contact interaction $(\mathrm{CI})$ between two initial-state quarks and two final-state leptons $(q q \ell \ell)$. Unlike resonance models, which require sufficient energy to produce the new gauge boson, the presence of a new interaction in the non-resonant regime can be detected at a much lower energy.

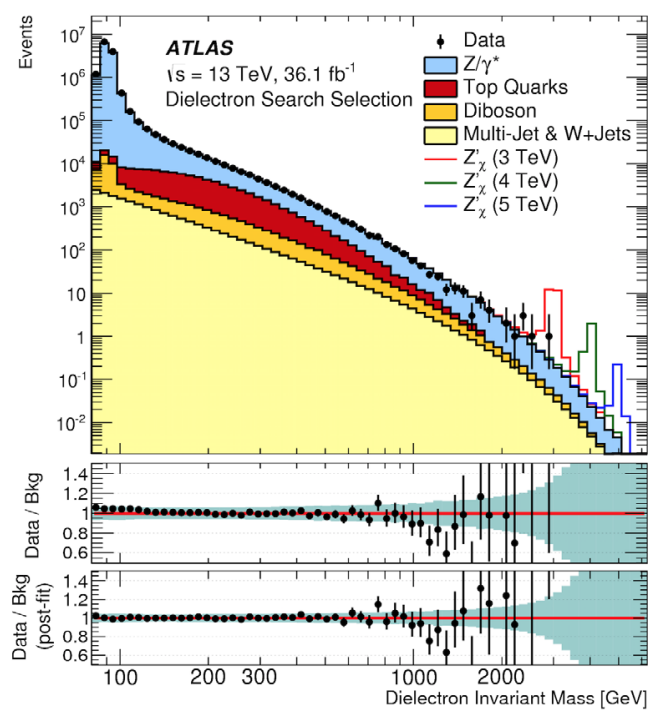

Figure 1. Distribution of dielectron reconstructed invariant mass $\left(m_{\ell \ell}\right)$ after selection, for data and the SM background estimates as well as their ratio before and after marginalisation. Selected $Z_{\chi}^{\prime}$ signals with a pole mass of 3,4 and $5 \mathrm{TeV}$ are overlaid. The bin width of the distributions is constant in $\log \left(m_{\ell \ell}\right)$ and the shaded band in the lower panels illustrates the total systematic uncertainty. The data points are shown together with their statistical uncertainty. From [6].

The $m_{\ell \ell}$ distributions, such as the one shown in Fig. 1 for dielectrons, are thoroughly examined for a resonant or non-resonant new physics excess using two different methods. The signal search and limit setting rely on a likelihood function, dependent on the parameter of interest, such as the signal cross-section, signal strength, coupling constant or the contact interaction scale. The data, scrutinised using statistical tests, show no significant excesses, so limits are set in the aforementioned parameters on resonant and non-resonant new physics models, as well as on generic resonances.

Such an example is presented in Fig. 2, where upper limits on the production cross section times branching fraction $(\sigma \mathcal{B})$ for $Z^{\prime}$ bosons are set. The weakening in sensitivity above a pole mass of $\sim 3.5 \mathrm{TeV}$ is mainly due to the combined effect of a rapidly falling signal cross section as the kinematic limit is approached, with an increasing proportion of the signal being produced off-shell in the lowmass tail, and the natural width of the resonance.

Lower limits are set at $95 \%$ confidence level (CL) on the energy scale $\Lambda$, for the LL, LR, RL, and RR Contact Interaction model. Both the constructive and destructive interference scenarios are explored, as well as priors of $1 / \Lambda^{2}$ and $1 / \Lambda^{4}$. Limits are presented for the combined dilepton channel in Fig. 3 using a $1 / \Lambda^{2}$ prior. 


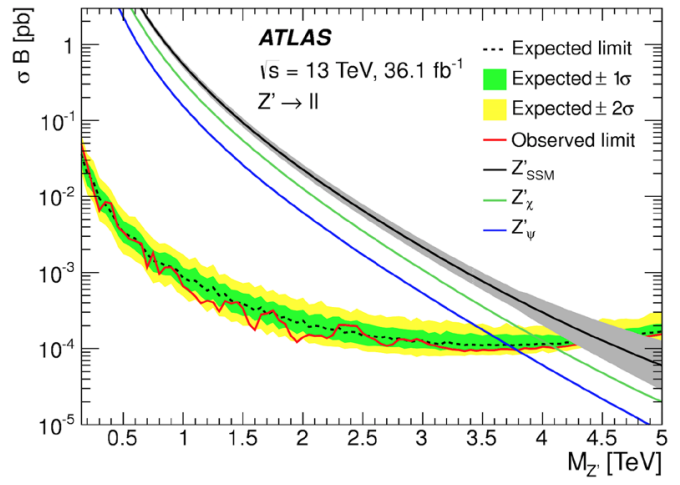

Figure 2. Upper 95\% CL limits on the $Z^{\prime}$ production $\sigma \mathcal{B}$ to two leptons of a single flavour as a function of $Z^{\prime}$ pole mass $\left(M_{Z^{\prime}}\right)$. Results are shown for the combined dilepton channel. The signals theoretical uncertainties are shown as a band on the $Z_{\text {SSM }}^{\prime}$ theory line for illustration purposes, but are not included in the $\sigma \mathcal{B}$ limit calculation. From [6].

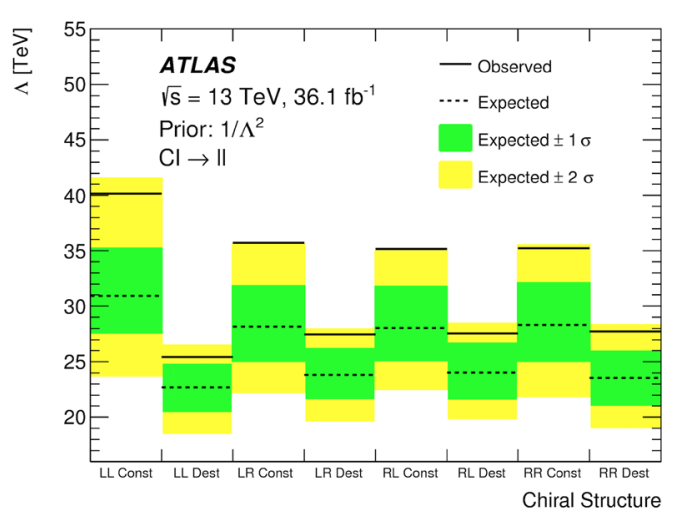

Figure 3. Lower limits on the energy scale $\Lambda$ at $95 \%$ CL, for the Contact Interaction model with constructive (const) and destructive (dest) interference, and all considered chiral structures with left-handed (L) and right-handed $(\mathrm{R})$ couplings. Results are shown for the combined dilepton channel. From [6].

In conclusion, the highest invariant mass event in this analysis is found at $2.90 \mathrm{TeV}$ in the dielectron channel, and $1.99 \mathrm{TeV}$ in the dimuon channel. The observed dilepton invariant mass spectrum is consistent with the SM prediction, within systematic and statistical uncertainties. Among a choice of different models, the data are interpreted in terms of resonant spin- $1 Z^{\prime}$ gauge boson production and non-resonant $q q \ell \ell$ contact interactions. For the resonant interpretation, upper limits are set on the cross-section times branching ratio for a spin-1 $Z^{\prime}$ gauge boson. The resulting $95 \%$ CL lower mass limits are $4.5 \mathrm{TeV}$ for the $Z_{\mathrm{SSM}}^{\prime}, 4.1 \mathrm{TeV}$ for the $Z_{\chi}^{\prime}$, and $3.8 \mathrm{TeV}$ for the $Z_{\psi}^{\prime}$. Other $\mathrm{E}_{6} Z^{\prime}$ models are also constrained in the range between those quoted for the $Z_{\chi}^{\prime}$ and $Z_{\psi}^{\prime}$. Lower mass limits are also set on the Minimal $Z^{\prime}$ model, up to $4.1 \mathrm{TeV}$ for the $Z_{3 R}^{\prime}$, and $4.2 \mathrm{TeV}$ for the $Z_{\mathrm{B}-\mathrm{L}}$. Generic $Z^{\prime}$ crosssection limits are also provided for a range of true signal widths. The lower limits on the energy scale

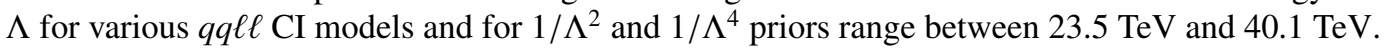

\subsection{Diphotons}

Searches for new phenomena in high-mass diphoton final states with ATLAS are presented in Ref. [11] using proton-proton collision data corresponding to an integrated luminosity of $36.7 \mathrm{fb}^{-1}$ recorded in 2015 and 2016 at $\sqrt{s}=13 \mathrm{TeV}$. Analyses optimised for the search for spin-0 resonances with masses above $200 \mathrm{GeV}$, for spin-2 resonances predicted by the Randall-Sundrum model [7] with masses above $500 \mathrm{GeV}$, and for non-resonant Kaluza-Klein (KK) graviton signals in the Arkani-HamedDimopoulos-Dvali (ADD) scenario [12] were performed.

The data were found to be consistent with the SM background expectation, as shown in Fig. 4. In the combined dataset, the largest local deviation from the background-only hypothesis for the spin0 (spin-2) resonance search was $2.6 \sigma(3.0 \sigma)$ for a mass near $730 \mathrm{GeV}$ and narrow width (mass near $708 \mathrm{GeV}$ and $\left.k / \bar{M}_{\mathrm{Pl}}=0.3\right)$. The global significance of this excess is null $(0.8 \sigma)$ for the spin- 0 (spin-2) resonance search.

In the spin-0 resonance search, the observed 95\% CL upper limits on the fiducial $\sigma \mathcal{B}$ for a narrowwidth signal range from $11.4 \mathrm{fb}$ at $200 \mathrm{GeV}$ to about $0.1 \mathrm{fb}$ at $2700 \mathrm{GeV}$. In the spin-2 resonance 


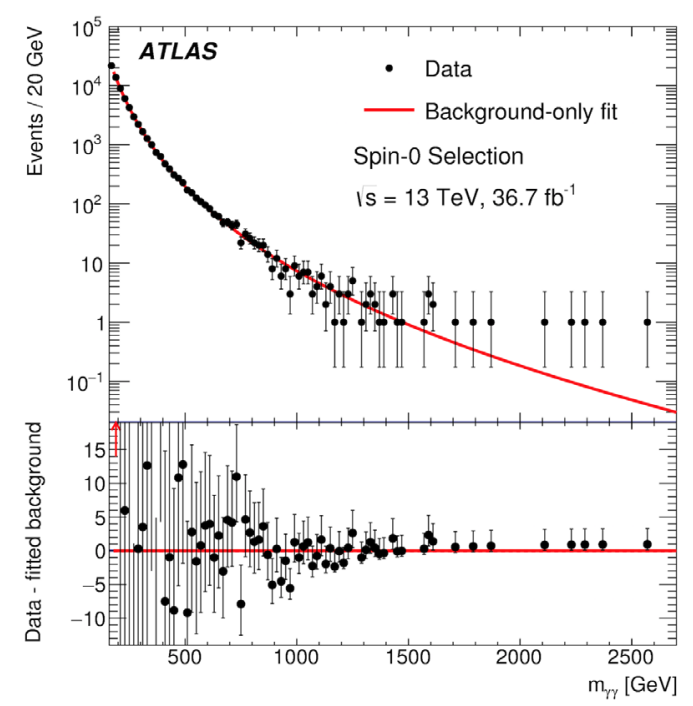

Figure 4. Distributions of the diphoton invariant mass for events passing the spin- 0 selection, with the background-only fit superimposed. The arrow in the lower panel indicate a difference between the data and the fit outside the range by $>1 \sigma$. There is no data event with $m_{\gamma \gamma}>2700 \mathrm{GeV}$. From [11].

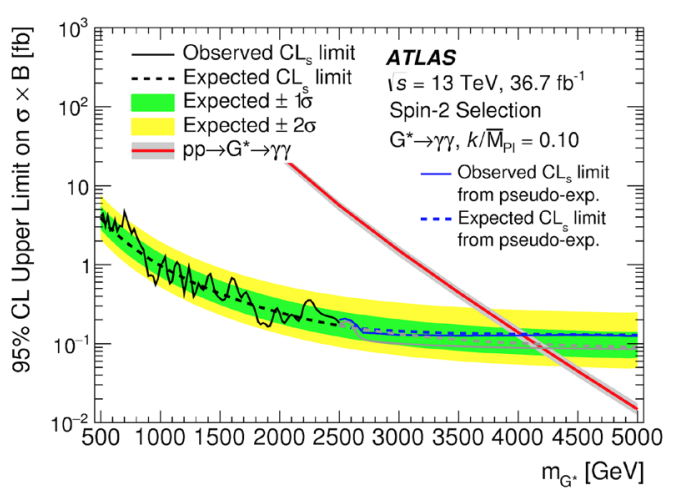

Figure 5. Upper limits on the $\sigma \mathcal{B}$ to two photons at $\sqrt{s}=13 \mathrm{TeV}$ of the lightest KK graviton as a function of its mass $m_{G^{*}}$ for $k / \bar{M}_{\mathrm{Pl}}=0.1$. For $m_{G^{*}}>$ $2500 \mathrm{GeV}$, the observed and expected limits are determined with pseudo-experiments shown by the blue solid and dashed lines, respectively. Predictions are shown for the RS1 model, where the grey shaded band represents the PDF uncertainty. From [11].

search, the observed limits on the cross section times branching ratio for $k / \bar{M}_{\mathrm{Pl}}=0.1$ range from $4.6 \mathrm{fb}$ to about $0.1 \mathrm{fb}$ for a KK graviton mass between $500 \mathrm{GeV}$ and $5000 \mathrm{GeV}$. The RS1 model with $k / \bar{M}_{\mathrm{Pl}}=0.1$ is excluded below $m_{G^{*}}=4.1 \mathrm{TeV}$, as presented in Fig. 5 .

In the ADD scenario, lower limits between 5.7 TeV and 8.6 TeV are set on the ultraviolet cutoff scale $M_{S}$, depending on the number of extra dimensions and the theoretical formalism used.

\subsection{Dibosons}

Diboson resonances are predicted in several extensions to the SM, such as composite Higgs models, warped extra dimensions, models with an extended Higgs sector and Grand Unified Theories. Searches have been conducted at the LHC in different decay modes, however no evidence of such resonances has been observed so far. The results can be expressed as limits in a simplified model which incorporates a Heavy Vector Triplet (HVT) of bosons [13].

\subsubsection{Resonances decaying to $V H \rightarrow q \bar{q} b \bar{b}$}

A search for resonances decaying to a $W$ or $Z$ boson and a Higgs boson has been carried out by ATLAS in the $q \bar{q}^{\left({ }^{\prime}\right)} b \bar{b}$ channel with $36.1 \mathrm{fb}^{-1}$ of $p p$ collision data. Both the vector and the Higgs boson candidates are reconstructed using large-radius jets, and jet mass and substructure observables are used to tag $W, Z$ and Higgs boson candidates and suppress the dominant multijet background. In addition, small radius $b$-tagged track jets ghost-associated to the large- $R$ jets are exploited to select the Higgs boson candidate jet. The data are in agreement with the SM expectations, with the largest 
excess observed at $m_{J J} \simeq 3.0 \mathrm{TeV}$ in the $Z H$ channel with a local (global) significance of $3.3 \sigma(2.1 \sigma)$. Upper limits on the production cross section times $\mathcal{B}(H \rightarrow b \bar{b})$ are set for resonance masses in the range between 1.1 and $3.8 \mathrm{TeV}$ with values ranging from $83 \mathrm{fb}$ to $1.6 \mathrm{fb}$ and $77 \mathrm{fb}$ to $1.1 \mathrm{fb}$ at $95 \%$ $\mathrm{CL}$ for $W H$ and $Z H$ resonances, respectively. The corresponding excluded HVT Model $B$ signal mass ranges are $1.1-2.5 \mathrm{TeV}$ for $W H$ resonances, and $1.1-2.6 \mathrm{TeV}$ for $Z H$ resonances. In addition, limits on the couplings of new heavy vector bosons to bosons, $g_{V} c_{H}$, and to fermions, $g^{2} c_{F} / g_{V}$, are derived as shown in Fig. 6.

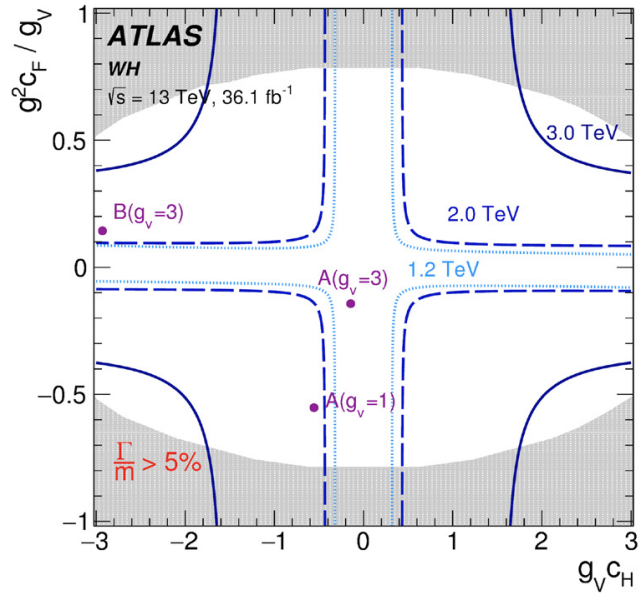

Figure 6. Limits in the $g^{2} c_{F} / g_{V}$ vs. $g_{V} c_{H}$ plane for several resonance masses for the $W H$ channel. Areas outside the curves are excluded. Coupling values for $\Gamma / m>5 \%$ may not be well described by the narrow-width approximation. From [14].

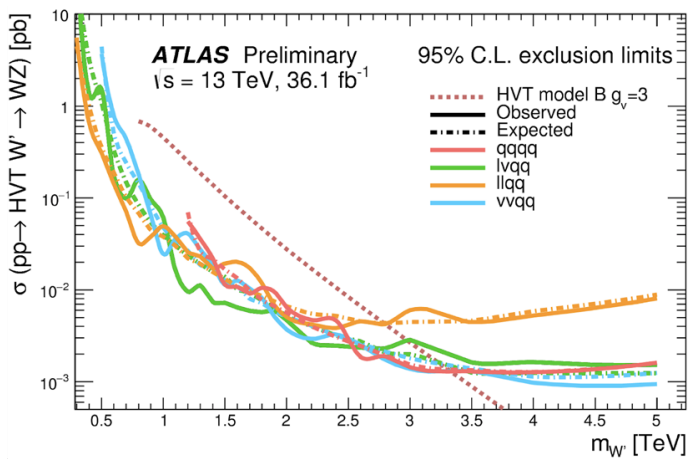

Figure 7. Expected and observed limits on the cross section times branching fraction to $W Z$ for a new heavy vector boson $W^{\prime}$ at $\sqrt{s}=13 \mathrm{TeV}$. The different limit curves correspond to different decay modes for the $W$ and $Z$ bosons. From [16].

\subsubsection{Resonances decaying to $W V \rightarrow \ell v q q$}

Another ATLAS analysis presented here discusses resonant $W W$ and $W Z$ production in semileptonic $(\ell v q q)$ final states using $36.1 \mathrm{fb}^{-1}$ of data collected at $\sqrt{s}=13 \mathrm{TeV}$ [15]. The analysis is carried out in different kinematic topologies according to the hadronically decaying $W / Z$ boson reconstruction. The data are compatible with the SM background hypothesis and no significant excess has been found. Limits on the production cross section are obtained as a function of the resonance mass for models predicting a narrow scalar boson, an HVT and a RS graviton, $G_{K K}$. Two different production modes are considered, the vector boson fusion and the gluon-gluon or quark-quark fusion (Drell-Yan, DY), and independent limits are set. Masses below 2750 (3090) GeV for HVT WW Model A (B), 2820 (2980) GeV for HVT $W Z$ Model $A(B)$, and $1760 \mathrm{GeV}$ for RS $G_{K K}$ signals with $k / \bar{M}_{\mathrm{Pl}}=1.0$ produced via gluon-gluon fusion are excluded at $95 \% \mathrm{CL}$. A summary of exclusion curves for HVT $W Z$ in the DY category is given in Fig. 7 for this analysis and for other searches in different channels.

\subsection{Vector-like quarks}

Naturalness arguments require that quadratic divergences arising from radiative corrections to the Higgs boson mass are cancelled by a new mechanism to avoid fine-tuning. Little Higgs and Composite 
Higgs models introduce a spontaneously broken global symmetry, with the Higgs boson emerging as a pseudo Nambu-Goldstone boson. These latter models predict the existence of vector-like quarks (VLQs), defined as colour-triplet spin-1/2 fermions whose left- and right-handed chiral components have the same transformation properties under the weak-isospin SU(2) gauge group. Depending on the model, vector-like quarks are produced in SU(2) singlets, doublets or triplets of flavours $T, B, X$ or $Y$, in which the first two have the same charge as the SM top and $b$ quarks while the vector-like $Y$ and $X$ quarks have charge $-4 / 3$ and $5 / 3$, respectively. VLQs are expected to couple preferentially to third-generation quarks and can have flavour-changing neutral-current decays.

ATLAS has performed a series of analyses targetting VLQs [16]. Here a search for the pair production of a heavy vector-like $T$ quark, based on $36.1 \mathrm{fb}^{-1}$ of $p p$ collisions at $\sqrt{s}=13 \mathrm{TeV}$ [17] is discussed. Data were analysed in the lepton-plus-jets final state and no significant deviation from the SM expectation was observed. Assuming a branching ratio $\mathcal{B}(T \rightarrow W b)=1$, the observed 95\% CL lower limit on the vector-like quark mass is $1350 \mathrm{GeV}$, as shown in Fig. 8, while for an SU(2) singlet $T$ quark, the observed mass limit is $1170 \mathrm{GeV}$.

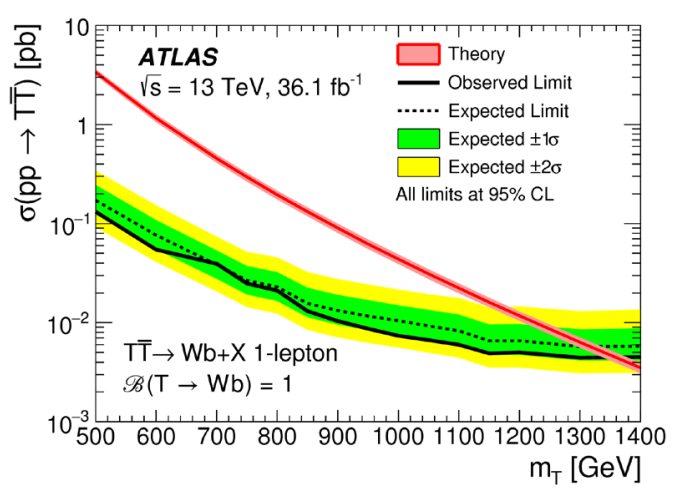

Figure 8. Expected (dashed black line) and observed (solid black line) upper limits at the $95 \% \mathrm{CL}$ on the $T \bar{T}$ cross-section as a function of $T$ quark mass assuming $\mathcal{B}(T \rightarrow W b)=1$. The green and yellow bands correspond to $\pm 1 \sigma$ and $\pm 2 \sigma$ around the expected limit. The thin red line and band show the theoretical prediction and its $\pm 1 \sigma$ uncertainty. From [17].

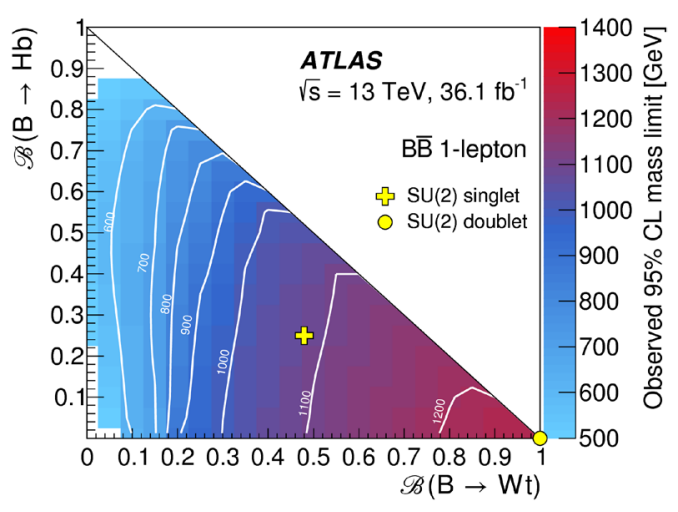

Figure 9. Observed 95\% CL lower limits on the mass of the $B$ quark in the branching-fraction plane of $\mathcal{B}(B \rightarrow W t)$ versus $\mathcal{B}(B \rightarrow H b)$. The markers indicate the branching ratios for the SU(2) singlet and doublet scenarios. The white regions are due to the limit falling below $500 \mathrm{GeV}$, the lowest simulated signal mass. From [17].

Assuming the $T$ quark can only decay to $\mathrm{Wb}, \mathrm{Zt}$ and $\mathrm{Ht}, 95 \% \mathrm{CL}$ lower limits are derived for various masses in the two-dimensional plane of $\mathcal{B}(T \rightarrow W b)$ versus $\mathcal{B}(T \rightarrow H t)$ [17]. This search is also reinterpreted to provide limits on $B$ quark masses. These are found to be $1250 \mathrm{GeV}$ assuming $100 \%$ branching ratio to $W t$ and $1080 \mathrm{GeV}$ under the $\mathrm{SU}(2)$ singlet $B$ quark scenario. These limits are found to be equally applicable to VLQ $Y$ quark and $X$ quark production, that decay to $W b$ and $W t$, respectively. Mass limits are also set as a function of the decay branching ratios $\mathcal{B}(B \rightarrow W t)$ versus $\mathcal{B}(B \rightarrow H b)$ assuming only the $B \rightarrow W t, B \rightarrow Z b$ and $B \rightarrow H b$ decay modes contribute (c.f. Fig. 9).

\section{Long-lived (SUSY) particles}

Supersymmetry (SUSY) is a popular extension of the SM which postulates the existence of a partner for every SM particle. These sparticles have the same quantum numbers as their SM counterparts but 
differ by one half-unit of spin. The loop corrections to the Higgs boson mass due to these sparticles are opposite to those of the SM particles thus providing a natural solution to the hierarchy problem. In addition, in $R$-parity conserving SUSY models, the lightest supersymmetric particle (LSP) offers itself as a good dark matter candidate subsequently explaining particular astrophysical observations.

In this Section, we present cases where at least one sparticle is long-lived but decays within the ATLAS detector volume. As we shall see, this imposes interesting experimental challenges on the pertinent object reconstruction and trigger. An overview of conventional SUSY searches is given in Ref. [18] with more details provided elsewhere on strong [19], electroweak (EW) [20] and thirdgeneration [21] production.

\subsection{Displaced vertices}

A search for massive, long-lived particles with decays giving rise to displaced multi-track vertices has been performed by ATLAS with $32.8 \mathrm{fb}^{-1}$ of $p p$ collisions at $\sqrt{s}=13 \mathrm{TeV}[22,23]$. A search for other displaced non-SUSY objects, namely lepton-jets, is described elsewhere [24]. The search is sensitive to models predicting events with significant $E_{\mathrm{T}}^{\text {miss }}$ and at least one displaced vertex with five or more tracks and a visible invariant mass $m_{\mathrm{DV}}$ greater than $10 \mathrm{GeV}$. Such models include Hidden Valley models, $R$-parity violating SUSY and Split Supersymmetry [25]. As benchmark scenario, a simplified topology of a long-lived $\tilde{g}$ (R-hadron) decaying into two quarks (jets) and the $\tilde{\chi}_{1}^{0}$ LSP is considered.

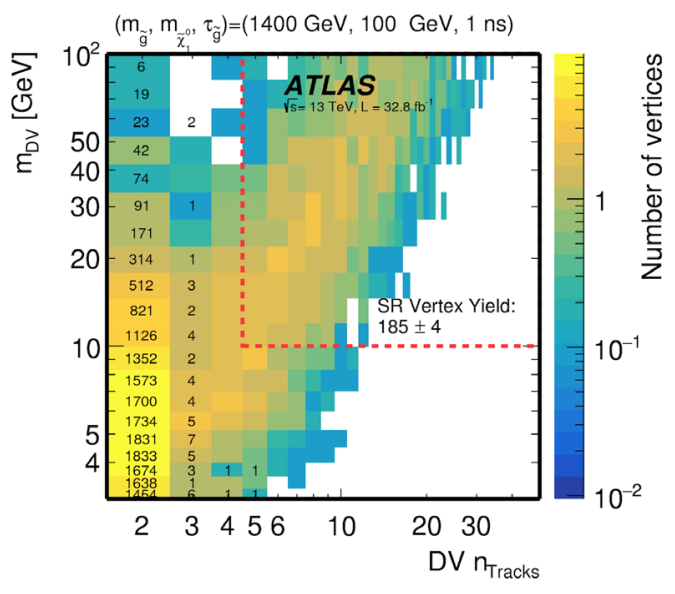

Figure 10. Two-dimensional distribution of $m_{\mathrm{DV}}$ and track multiplicity for DVs in events that satisfy all signal region event selection criteria. Bin numbers correspond to the observations in data, while the colour-representation shows example distribution for an R-hadron signal used as benchmark model in this search. The dashed line represents the boundary of the signal region requirements, and the expected signal yield in this region is shown. From [23].

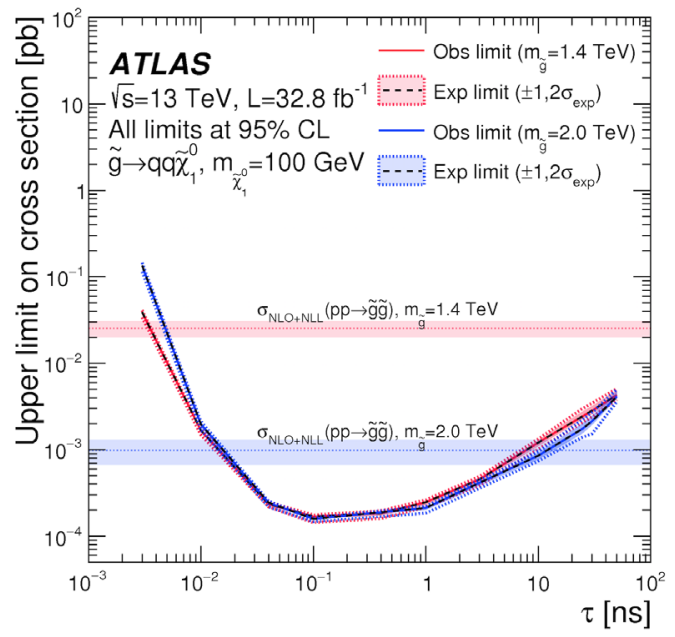

Figure 11. Upper 95\% CL limits on the signal cross section for $m_{\tilde{g}}=1.4 \mathrm{TeV}$ and $m_{\tilde{g}}=2 \mathrm{TeV}$ as a function of lifetime $\tau$, for fixed $m_{\tilde{\chi}_{1}^{0}}=100 \mathrm{GeV}$. Horizontal lines denote the $\tilde{g} \tilde{g}$ production cross section for the same values of $m_{\tilde{g}}$, with uncertainties due to the renormalisation and factorisation scale and PDF uncertainties. The expected and observed limit contours coincide due to the signal region yield's high level of agreement with expectation. From [23]. 
With an expected background of $0.02_{-0.01}^{+0.02}$ events, no events in the data sample were observed in the signal region, as seen in Fig. 10. With results consistent with the background-only hypothesis, exclusion limits are derived for models predicting the existence of such particles reaching roughly $m_{\tilde{g}}=2000 \mathrm{GeV}$ to $2370 \mathrm{GeV}$ for $m_{\tilde{\chi}_{1}^{0}}=100 \mathrm{GeV}$ and gluino lifetimes between 0.02 and $10 \mathrm{~ns}$, as shown in Fig. 11. For a fixed gluino-neutralino mass difference of $\Delta m=100 \mathrm{GeV}$, exclusion limits reach roughly $m_{\tilde{g}}=1550 \mathrm{GeV}$ to $1820 \mathrm{GeV}$ for gluino lifetimes between 0.02 and $4 \mathrm{~ns}$.

\subsection{Disappearing tracks}

A new search for long-lived charginos yielding a pixel tracklet signature has been performed based on $p p$ collision data at $\sqrt{s}=13 \mathrm{TeV}$ collected by ATLAS in 2015 and 2016, corresponding to an integrated luminosity of $36.1 \mathrm{fb}^{-1}[26,27]$. Tracklets having associated hits only in the pixel detector are used to improve significantly the sensitivity for short chargino lifetimes. By using pixel tracklets rather than standard tracks, the reconstruction efficiency is improved significantly for charginos decaying at radii shorter than $300 \mathrm{~mm}$, as evidenced in Fig. 12. The search targets decays of long-lived charginos that decay in the Pixel tracker to an (invisible) $\tilde{\chi}_{1}^{0}$ and a soft — hence undetected — charged pion. Such a case is typical of a wino LSP, where the lightest chargino and the LSP are nearly massdegenerate. Anomaly Mediated Supersymmetry Breaking (AMSB) scenarios [28] naturally predict such a pure wino LSP, with a mass splitting between the lightest chargino and the LSP to be around $160 \mathrm{MeV}$, corresponding to a chargino lifetime of about $0.2 \mathrm{~ns}$.

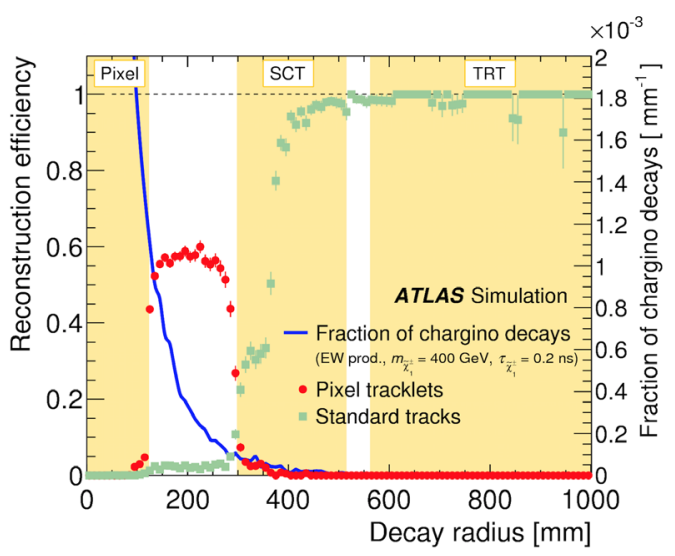

Figure 12. Chargino reconstruction efficiency as a function of decay radius. The distribution of the decay radius for charginos with a lifetime of $0.2 \mathrm{~ns}$ is shown in blue. The reconstruction efficiency of pixel tracklets before applying the fake-rejection criteria is shown in red, while that obtained with the standard tracking algorithm is shown in green. The yellow shaded regions correspond to the coverage of each detector. From [27].

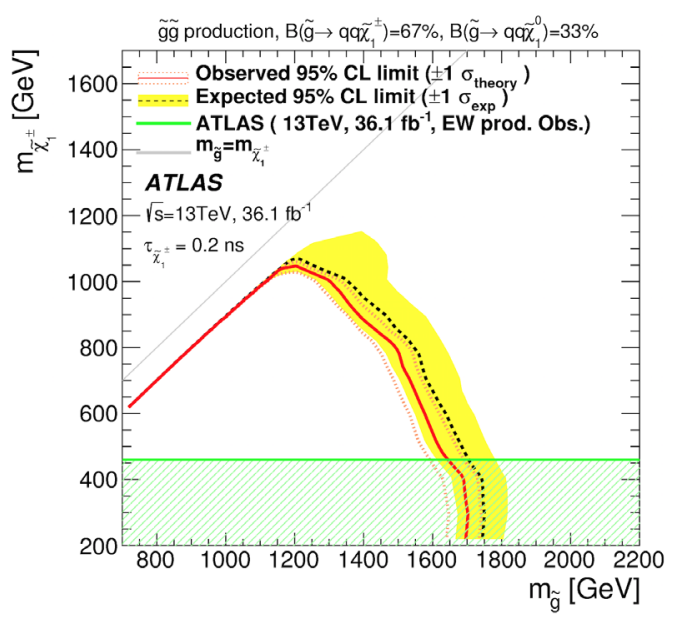

Figure 13. Exclusion limit at $95 \% \mathrm{CL}$ for the strongproduction channel in terms of $\tilde{g}$ and $\tilde{\chi}_{1}^{ \pm}$masses for a $\tilde{\chi}_{1}^{ \pm}$lifetime of $0.2 \mathrm{~ns}$. The yellow band shows the $1 \sigma$ uncertainty of the expected limit around its median (dashed line). The red line shows the observed limit and the orange band around it shows the $\pm 1 \sigma$ theoretical uncertainty on the cross section. Observed limits in the EW production search are shown as a green shaded region. From [27].

The $p_{\mathrm{T}}$ distribution of the pixel tracklets was found to be consistent with the background expectation. A lower limit on $m_{\tilde{\chi}_{1}^{ \pm}}$for electroweak production of long-lived charginos for a proper lifetime of 
$0.2 \mathrm{~ns}$, corresponding to a mass-splitting of $160 \mathrm{MeV}$, in the pure-wino LSP model is set at $430 \mathrm{GeV}$ at $95 \%$ CL. The case of charginos produced in the decay cascade of pair-produced gluinos is demonstrated in Fig. 13. Gluino masses below $1.6 \mathrm{TeV}$ are excluded assuming a chargino mass of $430 \mathrm{GeV}$, and below $1.05 \mathrm{TeV}$ for compressed spectra with mass difference between the gluino and the chargino of $200 \mathrm{GeV}$ for a proper lifetime of $0.2 \mathrm{~ns}$.

\section{Summary and outlook}

The Standard Model inherent limitations demand the presence of Physics beyond it, in a way that it is extended and complemented. ATLAS has searched for physics BSM at TeV scale in a variety of signatures inspired by a multitude of theoretical scenarios. No significant deviation from SM expectations has been observed so far. Nonetheless new data collected during the LHC Run 2 in 2017 and beyond may reveal hints of New Physics. ATLAS is well-prepared to make the most of them with analyses continuously improved with new trigger and/or reconstruction techniques and analysis channels.

An up-to-date account of the results from ATLAS searches for SUSY and beyond-SUSY is summarised in a tabulated form in Refs. [29] and [16], respectively.

\section{Acknowledgments}

The author is grateful to the ICNFP2017 organisers for the kind invitation to present this talk. She acknowledges support by the Spanish MINEICO under the project FPA2015-65652-C4-1-R, by the Generalitat Valenciana through the Excellence Project PROMETEO/2017/033, by the Severo Ochoa Excellence Centre Project SEV-2014-0398, and by a 2017 Leonardo Grant for Researchers and Cultural Creators, BBVA Foundation.

\section{References}

[1] L. Evans and P. Bryant, JINST 3, S08001 (2008).

[2] ATLAS Collaboration, JINST 3, S08003 (2008).

[3] P. Bruckman de Renstrom, "Search for neutral and charged BSM Higgs Bosons with the ATLAS detector," these proceedings.

[4] ATLAS Collaboration, New J. Phys. 18, no. 9, 093016 (2016) [arXiv:1605.06035 [hep-ex]].

[5] Y. Rozen, "Collider searches for dark matter," these proceedings.

[6] ATLAS Collaboration, JHEP 1710, 182 (2017) [arXiv:1707.02424 [hep-ex]].

[7] L. Randall and R. Sundrum, Phys. Rev. Lett. 83, 3370 (1999) [hep-ph/9905221].

[8] P. Meade and L. Randall, JHEP 0805, 003 (2008) [arXiv:0708.3017 [hep-ph]].

[9] M. V. Chizhov, V. A. Bednyakov and J. A. Budagov, Phys. Atom. Nucl. 71, 2096 (2008) [arXiv:0801.4235 [hep-ph]].

[10] F. Sannino and K. Tuominen, Phys. Rev. D 71, 051901 (2005) [hep-ph/0405209].

[11] ATLAS Collaboration, Phys. Lett. B 775, 105 (2017) [arXiv:1707.04147 [hep-ex]].

[12] N. Arkani-Hamed, S. Dimopoulos and G. R. Dvali, Phys. Lett. B 429, 263 (1998) [hep$\mathrm{ph} / 9803315]$.

[13] D. Pappadopulo, A. Thamm, R. Torre and A. Wulzer, JHEP 1409, 060 (2014) [arXiv:1402.4431 [hep-ph]];

J. de Blas, J. M. Lizana and M. Perez-Victoria, JHEP 1301, 166 (2013) [arXiv:1211.2229 [hep$\mathrm{ph}]$. 
[14] ATLAS Collaboration, Phys. Lett. B 774, 494 (2017) [arXiv:1707.06958 [hep-ex]].

[15] ATLAS Collaboration, "Search for $W W / W Z$ resonance production in $\ell v q q$ final states in $p p$ collisions at $\sqrt{s}=13 \mathrm{TeV}$ with the ATLAS detector," ATLAS-CONF-2017-051 (2017), http://cds.cern.ch/record/2273867.

[16] ATLAS Exotics Summary Plots web page: https://atlas.web.cern.ch/Atlas/GROUPS/PHYSICS/CombinedSummaryPlots/EXOTICS/

[17] ATLAS Collaboration, JHEP 1710, 141 (2017) [arXiv:1707.03347 [hep-ex]].

[18] A. Sopczak, "SUSY (ATLAS)," these proceedings.

[19] S. Adachi, "Inclusive searches for squarks and gluinos with the ATLAS detector," these proceedings.

[20] A. Kourkoumeli-Charalampidi, "Searches for electroweak production of supersymmetric gauginos and sleptons with the ATLAS detector," these proceedings.

[21] N. Koehler, "Searches for direct pair production of third generation squarks with the ATLAS detector," these proceedings.

[22] ATLAS collaboration, "Search for long-lived, massive particles in events with displaced vertices and missing transverse momentum in $13 \mathrm{TeV} p p$ collisions with the ATLAS detector," ATLASCONF-2017-026 (2017), http://cds.cern.ch/record/2258161.

[23] ATLAS Collaboration, "Search for long-lived, massive particles in events with displaced vertices and missing transverse momentum in $\sqrt{s}=13 \mathrm{TeV} p p$ collisions with the ATLAS detector," arXiv: 1710.04901 [hep-ex].

[24] C. Sebastiani, "Search for displaced lepton jets with the ATLAS experiment," these proceedings.

[25] N. Arkani-Hamed and S. Dimopoulos, JHEP 0506, 073 (2005) [hep-th/0405159];

G. F. Giudice and A. Romanino, Nucl. Phys. B 699, 65 (2004) Erratum: [Nucl. Phys. B 706, 487 (2005)] [hep-ph/0406088].

[26] ATLAS collaboration, "Search for long-lived charginos based on a disappearing-track signature in $p p$ collisions at $\sqrt{s}=13 \mathrm{TeV}$ with the ATLAS detector," ATLAS-CONF-2017-017 (2017), http://cds.cern.ch/record/2258131.

[27] ATLAS collaboration, "Search for long-lived charginos based on a disappearing-track signature in $p p$ collisions at $\sqrt{s}=13 \mathrm{TeV}$ with the ATLAS detector," arXiv:1712.02118 [hep-ex].

[28] G. F. Giudice, M. A. Luty, H. Murayama and R. Rattazzi, JHEP 9812, 027 (1998) [hep$\mathrm{ph} / 9810442]$;

L. Randall and R. Sundrum, Nucl. Phys. B 557, 79 (1999) [hep-th/9810155].

[29] Summary plots from the ATLAS Supersymmetry physics group: https://atlas.web.cern.ch/Atlas/GROUPS/PHYSICS/CombinedSummaryPlots/SUSY/ 